\title{
Autopsy Findings of Coronary Artery Dissection Occurring during Coronary Angiography
}

\author{
Palmer RF and McLemore J* \\ Department of Pathology, Wake Forest School of Medicine, USA
}

*Corresponding author: McLemore J, Department of Pathology, Wake Forest

School of Medicine, USA.

Received Date: July 07, 2020

Published Date: July 20, 2020

\begin{abstract}
The authors present autopsy findings from a case of catheter-induced coronary artery dissection during angiography. This adverse outcome is an uncommon but potentially devastating complication of coronary catheterization. The report will describe the clinical course and autopsy of an elderly woman with multiple comorbidities who had a coronary artery dissection during a coronary angiography procedure.
\end{abstract}

\section{Case Report}

An elderly woman with a history of multiple myeloma, hypertension, and congestive heart failure presented to the hospital with complaints of increased dyspnea on exertion, orthopnea, and lower extremity edema. She had completed six rounds of lenalidomide, bortezomib, and dexamethasone therapy for her multiple myeloma with very good partial response. At the hospital, she received an echocardiogram, which demonstrated a left ventricular ejection fraction of $23 \%$ with severe global hypokinesis. A nuclear stress test demonstrated ischemia in the distribution of the right coronary artery. She opted for a heart catheterization procedure for further evaluation. While performing the woman's angiogram, the clinicians noted a dissection of the left main coronary artery precipitating a quick decompensation of the patient's status shortly after the procedure began. She was emergently intubated and placed on extracorporeal membrane oxygenation but was pronounced dead a short time later. An autopsy was requested by the woman's family to rule out avoidable errors made by the clinicians and by the clinicians to better understand the reasons for the coronary artery dissection. Autopsy findings included noticeable dilation of the left main, proximal left anterior descending, and proximal left circumflex coronary arteries (Figure 1). A small area of serosal hemorrhage involved the anterior aspect of the proximal left circumflex coronary arteries. Cross-sections of the affected segments of the coronary arteries showed medial dissection with separation of the intima and a portion of the media from the remaining wall (Figure 2). The segments of vessels affected by the dissection had varying degrees of mild stenosis from atheromatous plaques. The aorta, however, demonstrated severe diffuse atherosclerosis with calcification and ulceration, which imparts an increased risk of dissection during catheterization. Although the exact location of the intimal defect could not be located, a dissection beginning in the aorta near or at the left coronary ostium with propagation of dissection into the left main, left anterior descending, and left circumflex coronaries is likely. 


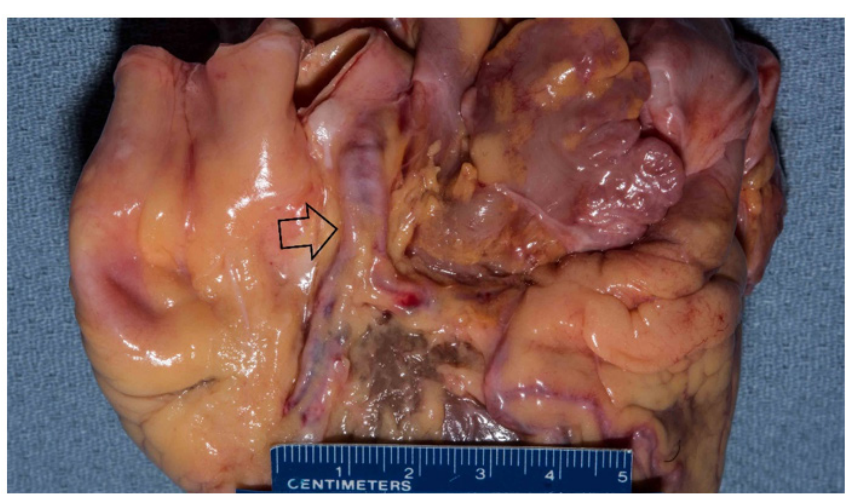

Figure 1: Dilated left main (arrow), left anterior descending, and left circumflex coronaries.

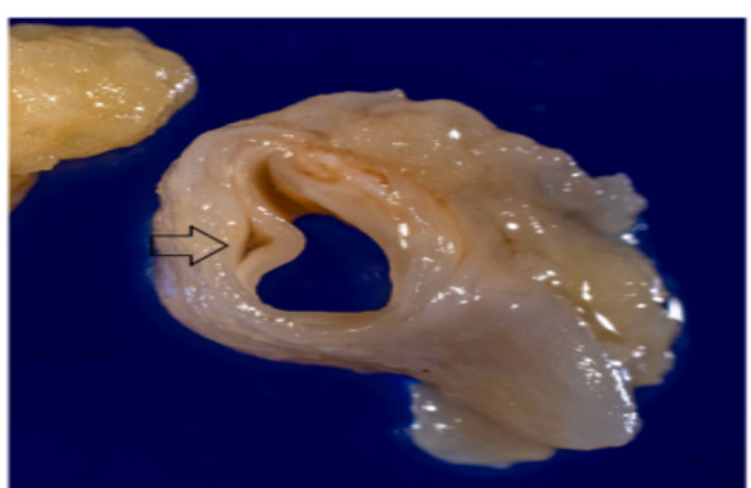

Figure 2: Cross-section of wall dissection

\section{Discussion}

Coronary artery angiography is a common, reliable, and safe procedure routinely performed to assess for the presence and extent of coronary artery atherosclerosis. Major complications from diagnostic cardiac catheterization are exceedingly rare, occurring in less than $2 \%$ of the general population. The mortality risk is even lower at $0.08 \%$ [1]. The incidence of catheter-induced iatrogenic coronary artery dissection is $<0.2 \%$ in the general population; however, the incidence of dissection increases in patients with a history of spontaneous coronary artery dissection [2]. Predisposing factors for spontaneous coronary artery dissection include both atherosclerotic and non-atherosclerotic causes. These factors contribute to the fragility of the coronary arterial walls, likely increasing the risk for both spontaneous and iatrogenic dissections [3]. The decedent was found to have severe atherosclerosis of the aorta, which was probably the site of the start of the dissection. Recent reports suggest non-atherosclerotic spontaneous coronary artery dissection predominately affects post-menopausal women with fibromuscular dysplasia and emotional stressors. In addition to post-menopausal status, systemic inflammatory conditions are also a risk factor [3].

Iatrogenic catheter-induced coronary artery dissection typically occurs in the setting of variant anatomy, catheter tip and/ or guidewire trauma, aggressive contrast injection, or angioplasty balloon overinflation [4]. Precautionary measures to reduce the risk of dissection include the appropriate selection of catheter type based on the patient's anatomy, careful handling of the catheter during procedure, and gradual injection of contrast media [5]. Left main coronary artery dissections producing symptomatic ischemia are currently managed with bail-out percutaneous coronary intervention (PCI) and coronary artery bypass grafting (CABG) [5]. Due to the time consuming process of transporting, prepping, and performing a CABG procedure, bail out PCI is the preferred initial management. CABG is performed when initial PCI treatment fails or in cases of extensive dissection and severe multivessel coronary artery disease in a hemodynamically stable patient [6]. The autopsy findings were important in providing better characterization of the dissection and in identifying possible risk factors that increased susceptibility for dissection during catheterization. The autopsy still plays an important role in the advancement of medicine and associated research. Unfortunately, in the United States, a recent removal of Centers for Medicare and Medicaid Services (CMS) regulation 482.22(d) eliminated the requirement that hospitals have an autopsy program to qualify for Medicare reimbursement [7]. This change in regulation will have a profound negative impact on medical education and research.

\section{Acknowledgement}

The authors wish to thank Mr. Kelly Pace, forensic photographer, Wake Forest School of Medicine, for the images. 


\section{Conflict of Interest}

\section{No conflict of interest.}

\section{References}

1. Tavakol M, Ashraf S, Brener SJ (2012) Risks and complications of coronary angiography: a comprehensive review. Global journal of health science 4(1): 65-93.

2. Prakash R, Starovoytov A, Heydari M, Mancini GB, Saw J (2016) Catheter-Induced Iatrogenic Coronary Artery Dissection in Patients With Spontaneous Coronary Artery Dissection. JACC. Cardiovascular interventions 9(17): 1851-1853.

3. Saw J, Aymong E, Sedlak T, Buller CE, Starovoytov A, et al. (2014) Spontaneous coronary artery dissection: association with predisposing arteriopathies and precipitating stressors and cardiovascular outcomes. Circulation. Cardiovascular interventions 7(5): 645-655.
4. Desai CK, Bhatnagar U, Stys A, Jonsson O (2017) Iatrogenic propagation of coronary dissection during diagnostic coronary angiography: an uncommon but important procedural consideration. BMJ case reports 2017: bcr2017222463.

5. Andreou AY, Avraamides PC, Andoniade T (2016) Iatrogenic left main coronary artery dissection: mind the catheter tip. Cardiovascular Medicine 19(10): 264-271.

6. Eshtehardi P, Adorjan P, Togni M, Hendrick Tevaearai, Rolf Vogel, et al. (2010) Iatrogenic left main coronary artery dissection: incidence, classification, management, and long term follow-up. Am Heart J 159(6): 1147-1153.

7. (2019) Medicare and Medicaid programs; regulatory provisions to promote program efficiency, transparency, and burden reduction; fire safety requirements for certain dialysis facilities; hospital and critical access hospital $(\mathrm{CAH})$ changes to promote innovation, flexibility, and improvement in patient care. Fed Regist 84: 51732-51834. 